Journal of

Applied

Crystallography

ISSN 0021-8898

Received 4 October 1999

Accepted 7 February 2000

(C) 2000 International Union of Crystallography

Printed in Great Britain - all rights reserved

\section{An introductory exercise in Fourier synthesis and structure-factor calculation for undergraduates, using an EXCEL workbook}

\begin{abstract}
Max R. Taylor
Department of Chemistry, The Flinders University of South Australia, GPO Box 2100, Adelaide, 5048, Australia. Correspondence e-mail: max.taylor@flinders.edu.au

An EXCEL (Microsoft Corp.) workbook has been configured to enable spreadsheet calculation of two-dimensional structure factors and Fourier syntheses. An exercise in which it can be used by undergraduate students to solve the structure of potassium bicarbonate in projection is described. The package is available from the internet.
\end{abstract}

\section{Introduction}

X-ray crystal structure analysis is now such a commonly used method of determining molecular structure that nearly every chemist or biologist will come across, and need to understand, the results of such an analysis in the course of their work. At Flinders University we include a topic in the regular undergraduate chemistry curriculum that aims to introduce and familiarize students with the terms commonly used when reporting the results of crystal structure analyses. They also come to understand what to look for when assessing the reliability of the analysis and derived parameters.

To bring the terms and techniques of crystal structure analysis to life, I set out to devise an exercise that all our students could take part in. While these days it is possible to provide sets of three-dimensional data to students, set them in front of a workstation, and using one of the standard packages, get them to 'solve' a structure, etc., for many students this 'black-box' approach adds little to their understanding. The logistics are also impossible for large groups of students.

I have found that a two-dimensional exercise, using a spreadsheet program on a PC, illustrates some of the principles involved and the language used in crystal structure solution. All of our students have had prior experience of using an EXCEL spreadsheet. The students experience about four lectures of theory prior to starting the exercise.

\section{The workbook}

Each student is provided with an EXCEL5 workbook configured with the basic requirements needed to calculate a two-dimensional Fourier synthesis and two-dimensional structure factors. The version currently used is restricted to the [100] projection of $P 2_{1} / c$ (symmetry $p 2 g g$ ), but it is readily converted to other symmetries or made more general.

The degree of difficulty of the exercise is easily varied by altering the amount of pre-programmed material that is provided to the student. Although in this application most of the crystallographic functions are provided, students can readily see the expressions that are being used in a way that they can easily understand. If more time is available, it is possible to give them the 'bare bones' and let them work it all out for themselves.

Structure factors. Contributions from each atom are calculated in columns and the student is left to combine these in an appropriate manner. Observed structure-factor data are provided on the absolute scale and isotropic displacement parameters for all atoms are usually provided.

Fourier syntheses. Three functions are provided for calculating Patterson, electron density and difference density maps. By suitable adjustment of spreadsheet cell size and font size, the maps are presented in an undistorted form and hard copies can be obtained. This gives the opportunity for some oldfashioned contouring and gives the student the now often lost thrill of 'seeing' the atoms develop in the structure.

Both of these functions are calculated point by point by macros. This is fast enough on a Pentium processor, and the more intuitively gifted students can recognize the logic of the calculations by reading the macros.

\section{The Example}

Although there are a great many structures that can be used, I have found that potassium bicarbonate (Pedersen, 1968) is an excellent example for a number of reasons. It has a convenient heavy atom and a small number of light atoms to locate. It crystallizes in $P 2_{1} / c$ with a short axis, $a=3.708 \AA$. It contains some of the elements of both organic and inorganic structural chemistry, viz. a trigonal planar carbon atom, covalent and ionic bonds, a hydrogen atom and a hydrogen bond, and a coordination polyhedron for the potassium atom. The carbonoxygen bonds can be used to illustrate resonance and the length relationships in single and double bonds. The $b$ axis is sufficiently short to enable the $x$ coordinates to be determined from the $h 0 l$ projection if desired. 


\section{The Procedure}

Starting with the basic workbook, students are instructed to first calculate $\sin \theta / \lambda$ and scattering-factor coefficients for all the reflections. The latter are calculated from stored polynomial coefficients. They may also be called upon to remove systematically absent reflections.

The students then proceed via the following sequence.

(i) Calculate a sharpened Patterson map and use it to determine the $y$ and $z$ coordinates of the potassium atom; alternatively, the potassium-atom coordinates may be provided.

(ii) Calculate structure factors and an electron density map phased from the potassium-atom contributions.

(ii) Contour the map to locate the carbon and oxygen atoms (Fig. 1).

(iv) Calculate a difference map and refine the positions of all the atoms, and eventually locate the hydrogen atom.

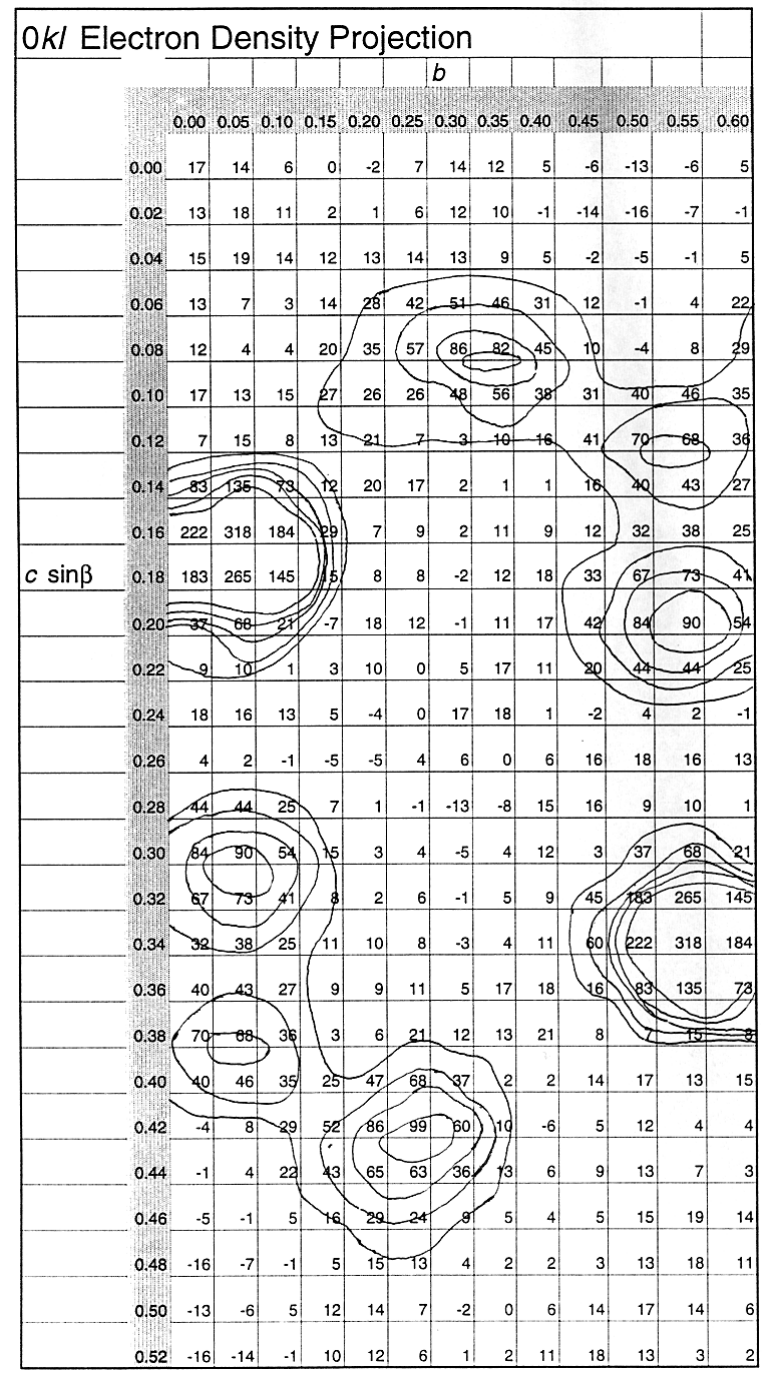

Figure 1

Part of the $0 \mathrm{kl}$ projection of the electron density of potassium bicarbonate produced by EXCEL. Fractional coordinates are provided and the numbers in the table are in units of $\times 10$ e $\AA^{-2}$.
At this stage, students can be given the $x$ coordinates, so that distances and angles can be calculated. They can be given a stereographic illustration of the unit-cell contents and be asked to write a description of the structure. This step is more than useful in the context of structural chemistry. Students are asked to submit a projection drawing of an asymmetric unit of the structure with its surroundings, as a symmetry exercise. The whole exercise takes $4-6 \mathrm{~h}$ to complete.

\section{Unique features}

During the refinement procedure it is not necessary to recalculate the whole difference map to check the positioning of an atom. The nature of spreadsheets is such that it is only necessary to refresh the small region that is of interest.

The 'what if' feature of spreadsheets means that every time the coordinate of an atom is changed, the structure factors are automatically recalculated and a new $R$ factor is available immediately. Some students very quickly realise that this means that they can refine the atomic positions by trial and error.

Although the use of the automatic graphing features of $E X C E L$ is deliberately not introduced, students who are more $E X C E L$ literate will want to use these functions to do the contouring for them. Some very nice plots can be obtained. I insist that each student contour the first map manually.

\section{Outcomes}

Use of the package may provide the following benefits.

An enthusiastic response.

An understanding of many of the terms used in the description of a crystal structure analysis.

A practical example with a real result that can be used in a hands-on mode on a familiar platform.

An appreciation of the precision that can be obtained in crystal structure analysis.

\section{Availability}

The workbook and notes have been deposited at the EPSRC funded CCP14 (Collaborative Computational Project No. 14 for Single Crystal and Powder Diffraction) website (http:// www.ccp14.ac.uk) (CCP14, 1999). The web address for this workbook and associated files is http://www.ccp14.ac.uk/ccp/ web-mirrors/max-taylor-fourier-spreadsheet/.

\section{References}

CCP14 (1999). Collaborative Computational Project No. 14 for Single Crystal and Powder Diffraction, CLRC Daresbury Laboratory, EPSRC, September, 1999. Chairman: Jeremy Karl Cockcroft. Secretary: Lachlan M. D. Cranswick. http://www.ccp14.ac.uk. Email: ccp14@ccp14.ac.uk.

Pedersen, B. (1968). Acta Cryst. B24, 478-480. 